\title{
BULLYING: COMPRENSIÓN DEL FENÓMENO DESDE OBSERVADORES/AS EN UN COLEGIO DE LA COMUNA DE CONCEPCIÓN, CHILE
}

\author{
BULLYING: COMPREHENSION OF THE PHENOMENON FROM \\ BYSTANDER STUDENT, IN A SCHOOL OF THE TOWN \\ OF CONCEPCIÓN, CHILE
}

Daniela Esquivel Suazo*, Cristian Segura Carrillo**

\section{Resumen}

El presente artículo forma parte de la investigación para obtener el grado de $\mathrm{Ma}$ gister en Intervención Familiar y tiene por objetivo, comprender la experiencia de Bullying, desde el significado otorgado por estudiantes que han desempeñado el rol de observador/a, pertenecientes a un Colegio de la comuna de Concepción. La investigación es de tipo cualitativa-interpretativa con enfoque fenomenológico. Para la interpretación de los resultados, se realizó un análisis de contenido mediante ATLAS ti v.8. Entre sus principales hallazgos; los/as estudiantes significan el fenómeno como una forma negativa de relacionarse entre pares, conducta que se encuentra internalizada en la vida escolar, al punto de encubrir las agresiones realizadas por compañeros/as, debido a las consecuencias sociales que implica la acción. Entre los factores que inciden en la forma de actuar de los/as entrevistados/as, se perciben influencias familiares y de los medios de comunicación. Respecto al fenómeno, se observa desconocimiento en cuanto a las consecuencias que genera el acoso escolar en sus diferentes actores. Aun cuando existe presencia del fenómeno, el clima escolar se percibe de forma positiva; sin embargo, se aprecian algunas fallas comunicacionales que favorecen la presencia del acoso escolar.

Palabras clave: Relaciones entre pares, acoso escolar, observadores/a de acoso, violencia, ambiente educacional.

*Trabajadora Social, Magister en Intervención Familiar, Universidad de Concepción, Concepción, Chile.

** Trabajador Social, Mg. en Trabajo Social y Políticas Sociales, Mg. en Docencia para la Educación Superior, Profesor Asistente Depto. Trabajo Social, Universidad de Concepción, Concepción, Chile, crisegurac@udec.cl. 


\begin{abstract}
This article is part of the research to obtain the degree of Master in Family Intervention and aims to understand the experience of Bullying, from the meaning given by students who have played the role of bystanders of a school in Concepción. The research has a qualitative and interpretative approach with a phenomenological focus. For the interpretation of the results, a content analysis was carried out using ATLAS ti v.8. Among its main findings, the students signify the phenomenon as a negative way of relating among peers, a behavior that is internalized in school life, to the point of covering up peer aggression, due to the social consequences that the action implies. Among the factors that influence the way in which the interviewees act, family and mass media influences are perceived. Regarding the phenomenon, there is a lack of knowledge regarding the consequences that school bullying generates in its different actors. Even when the phenomenon is present, the school climate is perceived positively; however, some communication failures are observed that facilitate the presence of bullying.
\end{abstract}

Keywords: Peer relationship, Bullying, Bystander to bullying, violence, Educational environment.

\title{
1. Introducción
}

$\mathrm{E}$

L BULlying es un fenómeno que ocurre desde hace años; no obstante, desde la década del 70 comenzó a ser estudiado por Olweus, quien se interesó en las relaciones entre estudiantes, acuñando el término bullying para referirse a: "cuando un estudiante es acosado o victimizado, estando expuesto de manera repetitiva a acciones negativas por parte de uno o más estudiantes" (Olweus, 1993, p2). Esta práctica, se caracteriza por la diferencia de poder que existe entre sus participantes, dando origen a una relación de desigualdad. Los efectos causados son varios, dependerán del rol que se realice durante la interacción violenta, en el caso de la víctima; puede desarrollar deficiente desempeño escolar, comportamiento retraído, tartamudeo, alteraciones alimentarias, trastornos obsesivos, sufrimiento y disminución del apetito (García y Ascencio, 2015). A nivel de salud mental; Ansiedad, fobia social, depresión y tendencias suicidas (Musalem y Castro, 2015). En el caso de los victimarios, hay probabilidad de 
inestabilidad emocional, angustia, irritabilidad, ansiedad, depresión y posibles trastornos psicopatológicos, consumo de drogas, problemas sociales, dificultad para convivir con otras personas, problemas para establecer relaciones y probables conductas delictivas en el futuro (Romero y Bravo, 2017; González, Mariaca y Arias, 2014). Por último, en los observadores; falta de sensibilidad frente al dolor ajeno y escasa solidaridad (Arroyave, 2012).

Debido a la magnitud de sus consecuencias, el acoso escolar es estudiado en diferentes países y contextos. Autores como Sánchez (2013) señala que las figuras de víctima y victimario no nacen desde lo particular, por el contrario, se construyen de forma social, por ello, en su investigación "El bullying como construcción social, más allá de las víctimas, los agresores y los testigos... La familia, los docentes y la sociedad" en Colombia, reafirma su mirada al concluir que este fenómeno es un problema que debe ser abordado por toda la comunidad educativa y que incluso la sociedad debe formar parte, ya que este sería un reflejo de cómo se encuentra hoy en día. En España, autoras como Pulido y Tarancón (2018) en su investigación "Opiniones y experiencias respecto al bullying: Estudio cualitativo en un Instituto de Educación Secundaria de la localidad de Albacete", mencionan que existe cierta desconfianza por parte de los/as estudiantes respecto al accionar del profesorado, por ello, prefieren la intervención de un agente externo (psicólogo/a) para tratar los casos de bullying, con el fin de resguardar el anonimato y para recibir un tratamiento que realmente ayude a los involucrados. Las diferentes perspectivas de un fenómeno, el hecho de que en una de las investigaciones se perciba como un problema social y en el otro como un problema patológico que debe requerir la intervención y tratamiento de un psicólogo, habla de la importancia cultural no solo a la hora de estudiar este tipo de prácticas, sino también para ser intervenidas.

Aun cuando se conocen los efectos del acoso escolar, los tipos, los actores y los factores que dan origen a la práctica, esta continúa y persiste en los establecimientos educacionales, por ello es preciso continuar estudiando el fenómeno y realizar esfuerzos para comprender esta práctica desde sus principales actores, es decir, los estudiantes y para esta investigación aquellos/as que hayan cumplido el rol de 
observador/a, ya que la importancia de este rol radica en el apoyo que le entregan a la víctima o al victimario, es decir, es la audiencia la que permite que la práctica continúe o se detenga.

Existen tres tipos de observadores: Observador activo, aquel que sirve como refuerzo del agresor, es decir, forma parte de la red de apoyo del victimario y avala las acciones que realiza, observando la agresión y animándola o incitándola a través de risas o gestos alentadores, estos no atacan de forma directa a la víctima. Observador Pasivo; aquel que se mantiene distante, ignorando la situación ocurrida, no toma partido por ningún rol, es decir, la víctima o el victimario; aun cuando no se involucra, su modo de actuar se considera como una aprobación silenciosa al acoso escolar. Por último, el observador Proactivo; realiza el rol de defensor, toma partido a favor de la víctima, sus estrategias son buscar ayuda en pares, profesores o adultos de la comunidad educativa con el fin de tratar de detener el acoso. Estos observadores son los que tienden a disminuir el daño emocional causado por el intimidador, puesto que su ayuda entrega bienestar y seguridad a la víctima (Salmivalli, 1999).

A través de los observadores, se pretende identificar los factores que intervienen en las conductas que desarrollan y en el rol que asumen (observadores/as pasivos, activos o proactivos). Para ello, se utilizaron 4 dimensiones que direccionaron la investigación: La experiencia de bullying, los mecanismos de acción, el clima escolar y aspectos culturales. Este último elemento responde a la necesidad de situar al sujeto participante para conocer de qué forma los patrones culturales influyen en la conducta que estos realizan.

\section{Teorías que permiten comprender el fenómeno}

\subsection{Teoría Ecológica: Clima escolar y cultura}

Desde la perspectiva ecológica, el ser humano se encuentra expuesto a diferentes estímulos que pueden alterar su conducta de forma permanente. Esto no siempre es positivo, por ello es importante considerar el contexto en su totalidad, puesto que determinará el comportamiento, 
aun cuando no se participe de forma directa en cada uno de ellos (Gifre y Guitart, 2013). De acuerdo con la teoría ecológica, el ser humano constantemente recibe influencias de diferentes ámbitos, ya sea el ámbito familiar o social, que incidirán en su forma de actuar y comportarse frente a la sociedad. Siguiendo la idea anterior, el ambiente ecológico es un "conjunto de estructuras seriadas y estructuradas en diferentes niveles" (Bronfenbrenner, 1987, p. 23), en donde cada uno de esos niveles contiene al otro. Estos niveles se denominan microsistema, mesosistema, exosistema y macrosistema. Bajo la perspectiva ecológica, la característica distintiva de los niveles consiste en su relación directa, es decir, cuando uno de los sistemas es alterado, este repercute en el funcionamiento de los demás.

El primer nivel, el microsistema es el más cercano al ser humano. Está constituido por la familia o por los sistemas más próximos, con los cuales establece relaciones significativas, en el caso de un niño, niña o adolescente aparte de la familia, se encuentra el colegio y el grupo de amigos. En este nivel, es posible establecer roles que determinarán cuáles serán las acciones que se realizarán dentro de un contexto. En el caso del colegio, los adolescentes asumen el rol de estudiantes, donde sus principales funciones están relacionadas con el aprendizaje y la interacción con otros, no obstante, dentro de las dinámicas escolares y en específico dentro de las interacciones aparece un fenómeno que perjudica el contexto escolar: El bullying e inclusive en él es posible encontrar roles (víctima, victimario, observador, etc.).

El segundo entorno más importante del microsistema es el colegio, corresponde a la siguiente esfera de socialización entre los/as jóvenes. En él, es posible establecer relaciones, afianzar lazos y poner a prueba los límites en cuanto al respeto por el otro (Gifre y Guitart, 2013), sin embargo, en algunos casos estos límites no son respetados, dando origen a malas prácticas que pueden perturbar el clima escolar.

El segundo sistema para analizar es el mesosistema. En este nivel dos o más sistemas se interrelacionan, su importancia radica en la eficacia de las interconexiones sociales entre los entornos, uno de los factores principales para lograr ese objetivo es la participación conjunta entre la comunicación y el conocimiento o información que se obtenga de cada entorno (Briceño, 2017). Bajo este enunciado, las relaciones 
entre los sistemas son esenciales para el desarrollo de las personas. En el caso del contexto escolar, en ocasiones se presentan vacíos en cuanto a la relación que existe entre docentes y padres, debido a la falta de tiempo o porque ambos sistemas se ven absorbidos por diversas tareas y preocupaciones, sin embargo, como la teoría lo señala, este tipo de situaciones no favorece el óptimo funcionamiento, por el contrario, genera consecuencias que en muchos casos pueden ser irreversibles.

Hasta el momento, se ha hablado de los entornos cercanos, como el microsistema y el mesosistema, sin embargo, quedan dos sistemas por abordar; el exosistema y el macrosistema. En primer lugar, el exosistema responde a "uno o más entornos que no incluyen a la persona en desarrollo como participante activo, pero en los que se producen hechos que afectan, o se ven afectados por lo que ocurre en ese entorno" (Bronfenbrenner, 1987, p. 261). El sistema escolar, la comunidad, los medios de comunicación, entre otros; forman parte de este sistema y lo importante de ellos es cómo afectan al individuo. En el caso del acoso escolar, llama la atención cómo aspectos de la cultura intervienen en él a través de diversos medios, por ejemplo: los medios de comunicación, en ellos es posible encontrar un sin fin de imágenes violentas o agresivas que de alguna u otra forma alteran la percepción de quien las ve, en algunos casos imitando las conductas violentas como si estas fueran permitidas o incluso normales cuando en realidad no lo son.

El último sistema es el macrosistema compuesto por "la cultura y el conjunto de valores e ideologías predominantes en el medio en el que se desarrolla la persona” (Briceño, 2017). En este sistema posee gran relevancia la cultura, su importancia radica en cómo influye en el individuo, ya sea en la vestimenta, la forma de comer o hablar, de una u otra forma determina el comportamiento de los individuos. Un ejemplo de ellos, son los programas para prevenir o intervenir el acoso escolar, cuando se replican programas en Latinoamérica que provienen de países europeos no funcionan si estos no se adaptan a la realidad latinoamericana, incluso entre estos países existen muchas diferencias, aun cuando forman parte del mismo continente.

En cuanto al macrosistema, su importancia radica en cómo afecta a los demás niveles, por ejemplo; hasta hace 50 décadas, el acoso escolar era un fenómeno que ocurría en las aulas de clases de niños, 
niñas y adolescentes y que no era estudiado en mayor profundidad. Sin embargo, desde que Olweus comenzó sus estudios y demostró las consecuencias que conlleva, este comenzó a ser investigado por más autores hasta que generó preocupación en la sociedad, dando lugar a programas de prevención y estrategias de intervención para reducir el bullying. De a poco, se generó un cambio cultural respecto a la violencia en los colegios que dio origen a las acciones que hoy en día responden a una problemática social.

\subsection{Teoria del Aprendizaje Social: Observadores y Familia}

Bandura (1976) señala que las personas no nacen con pautas de interacciones o reacciones, estas las aprenden a través de la relación con otros. De acuerdo con lo señalado por Bandura, el ser humano actuaría de acuerdo a las consecuencias que genera una acción, pueden tener un efecto positivo o negativo. En el caso del acoso escolar, los estudiantes llevan a cabo una conducta agresiva con la finalidad de intimidar a un estudiante, sin embargo, en este caso las consecuencias de la intimidación poseen dos lados: por uno, la satisfacción que genera al hostigador provocar daño, sin que esto le genere sentimiento de culpa (Ruiz, Riuró y Tesouro, 2015; Sánchez, 2013; Arango, 2014) y, por otro, se encuentran las consecuencias que genera la acción, es decir, un regaño, sanción o castigo. Por lo tanto, en el caso del victimario sus acciones demostrarían falta de empatía hacia el dolor ajeno y ausencia de un proceso reflexivo respecto de sus actos.

Bandura plantea que de las acciones o respuestas que realiza un sujeto resultan tres funciones que explican y detallan cómo se aprende a través de las consecuencias de un acto, ellas son: normativa (proporcionan información), motivacional (las consecuencias que advierten de sus respuestas) y reforzante (como una forma de regular las conductas aprendidas) (López y Guaimara, 2016). Estas respuestas, se vincularían con el accionar de los observadores al enfrentarse a situaciones de bullying. En cuanto a la función normativa, se aprende a través de la conciencia que se tiene de un acto. En el caso de los observadores, aprenden a callar o intervenir, de acuerdo a la información obtenida del ejemplo de otros. 
En cuanto a la función motivacional, esta hace referencia al origen de la conducta. El caso de los observadores, su origen depende de muchos factores, por ejemplo: Hazler (1996) señala que los observadores pasivos no actúan porque no saben cómo ayudar porque tienen miedo a convertirse en víctimas y porque piensan que podrían hacer las cosas mal, perjudicando aún más a la víctima. En el caso del observador activo, su origen proviene de la atracción que siente por el poder y autoridad que posee el agresor y en el caso de los observadores proactivos, su comportamiento proviene de visualizar a la víctima como una persona digna de ser ayudada, con el fin de favorecer las relaciones interpersonales (Cuevas y Marmolejo, 2016).

Por último, la función reforzante, regula la conducta aprendida a través de estímulos, en otras palabras, ensayo y error. Estos estímulos provienen de diversos contextos, no solo del contexto escolar, por ejemplo, si un estudiante se encuentra constantemente enfrentado a situaciones agresivas, existe una alta probabilidad de que en un futuro evite los escenarios violentos o en el caso contrario, puede desarrollar insensibilización ante imágenes violentas (Cuevas y Marmolejo, 2016). En el caso de los observadores que apoyan la agresión, aprenden que existe una relación directa entre apoyar al líder y no ser molestados, por ello apoyan las acciones que realiza el agresor.

En cuanto al origen del rol de observador, Cuevas y Marmolejo (2016) señalan que depende de muchos factores, entre ellos se encuentran los interpersonales, donde el estilo de crianza desarrollado por los padres es primordial. Por ejemplo, en el caso del observador proactivo, (aquel que apoya a la víctima) es asociado a ciertas características del individuo que le permiten generar una conexión con la víctima, entre ellas se encuentran la empatía y la asertividad. Estas características son aprendidas a través de los primeros cuidadores, ellos al establecer vínculos afectivos seguros, protectores y estables, otorgan al individuo la confianza necesaria para desarrollar acciones que le permitan defender o proteger a la víctima. Por el contrario, cuando los padres no imparten este tipo de prácticas y tampoco generan conciencia en los niños respecto al daño que sufre la víctima, surgen otras formas de reacción, como observar la acción sin prestar ayuda o aprobar la conducta del agresor (observador pasivo y activo). 
Siguiendo la idea anterior, la familia cumple un rol fundamental en el rol del observador, no tan solo mediante el desarrollo de habilidades sociales que les permitan interactuar con otros. Además, estos deben ser categóricos en cuanto al rechazo de conductas violentas y/o agresivas hacia otro compañero y señalar que el silencio u omisión de este tipo de acciones (acoso escolar) es igual de negativo que realizar la acción, debido a las consecuencias que genera en la víctima (Kivaprogram, 2013).

De acuerdo con las teorías señaladas con anterioridad, el bullying es un fenómeno que para ser intervenido requiere de la unidad de la comunidad educativa, por ello los programas creados para abordarlo tienen en consideración estos paradigmas. Uno de los programas más reconocidos a nivel mundial para intervenir el acoso escolar es el programa KIVA de origen finlandés. Sus objetivos son, en primer lugar, entregar información básica a los colegios sobre el acoso escolar y alternativas o soluciones para abordarlo, como una forma de comprometer a la comunidad escolar. El segundo objetivo, se centra en el grupo (curso) con la finalidad de terminar con la cultura del silencio, es decir, que los estudiantes hablen sobre las situaciones de acoso escolar que observan y entreguen su apoyo a la víctima. Para finalizar, el tercer objetivo se centra en los estudiantes afectados. Para ello, se realiza un seguimiento de los casos graves de violencia, comprobando que el acoso ha disminuido hasta su eliminación (Azofra, 2017; Mäkelä y Catalán, 2018). De esta forma, el programa se encarga de intervenir diferentes áreas, de manera individual prestando apoyo, realizando seguimiento a la víctima y de manera grupal para generar conciencia entre los/as estudiantes y denuncien los hechos de acoso escolar que observan, resaltando la importancia de no solo incluir a los/as estudiantes, sino que también a la comunidad educativa, en consecuencia, esta es una forma de realizar un cambio real.

Otro programa es WiSk, elaborado en la facultad de Psicología de la Universidad de Viena. El objetivo del programa es desarrollar habilidades sociales en la escuela e involucrar a toda la comunidad escolar para lograr su efectividad. Su desarrollo equivale a 4 partes: La primera consiste en formar un equipo de docentes que asuman la responsabilidad del programa, en segundo lugar aprobar un reglamento 
que será aplicado continuamente en la escuela, en tercer lugar diseñar un plan de acción para todo el colegio y, por último, promover reuniones de información a toda la comunidad escolar (padres, profesores y estudiantes), junto con vigilar de forma exhaustiva el patio y desplegar las acciones necesarias ante la presencia de acoso escolar (Spiel, Strohmeir y Atrias, 2010). En este programa es importante resaltar la responsabilidad de los docentes al asumir el plan de acción, pues en ellos recae la tarea de enseñar a los/a estudiantes nuevas formas de convivencia, que permiten mejorar el clima escolar.

Romera et al. (2017) en sus trabajos, propone medidas estratégicas para enfrentar el bullying y cyberacoso, entre ellas; la acción tutorial y mediación. Destaca que, si bien en los sistemas educativos prima el trabajo individualizado, resulta a la vez necesario desarrollar la competencia social, que les permita a los/as estudiantes adquirir habilidades para vincularse de manera sana con sus compañeros/as. Así, las tutorías ocuparían un rol fundamental, ya que se encargarían de desarrollar esta competencia social. En el caso de las mediaciones y los programas de ayuda entre iguales, promueven la participación de los/as estudiantes para que sean ellos mismos quienes encuentren formas adecuadas para resolver sus problemas.

Llama la atención en los programas revisados con anterioridad el carácter social y sistémico, en el sentido de que el mundo escolar posee diferentes sistemas, formado por: padres, estudiantes, profesores, entre otros. El problema surge cuando se presenta una falla, puesto que termina por alterar el funcionamiento de todo los sistemas, en el caso de la convivencia escolar cuando los/as estudiantes perciben desidia por parte de sus profesores, es decir, que a estos no les interesa la relación entre los/as estudiantes o lo que entre ellos pueda pasar, existen mayores probabilidades de que se presente un fenómeno como el acoso escolar (Ortega, Del Rey y Casas, 2013), de esta forma, aspectos como la comunicación suelen ser fundamentales. Es necesario mantener una comunicación abierta y honesta, entre todos aquellos que forman el sistema para tener una visión clara y completa del problema. En el caso de la presente investigación, resulta necesario obtener la opinión de los/as observadores para comprender su postura frente a un fenómeno que observan y que de alguna u otra forma los afecta. 


\section{Material y método}

La presente investigación fue de carácter cualitativa interpretativa con enfoque fenomenológico. Desde el punto de vista cualitativo, el estudio buscó trabajar de manera interactiva, para identificar las diferencias lógicas de los actores, que surgirán de la temática, para posteriormente interpretarlas desde la lógica de los mismos participantes $(\mathrm{Ga}-$ leano, 2004). El carácter fenomenológico de la investigación pretendió comprender cómo significan los/as observadores/as el acoso escolar, a través de su propio discurso. En este tipo de enfoque, la realidad que importa es aquella que las personas consideran importantes (Galeano, 2004).

\section{Diseño Muestral/participantes}

El tipo de muestra corresponde a un muestreo teórico-intencional, es decir, se seleccionaron aquellos casos que respondieron a las necesidades de la investigación, en esta ocasión la muestra se encuentra conformada por siete estudiantes (cinco mujeres y dos hombres) observadores de bullying (Otzen y Manterola, 2017), ya que ellos/as son uno de los/as principales involucrados/as en el acoso escolar. Durante el proceso de selección, debió ser eliminada de la muestra una de las participantes porque presentaba experiencias previas de acoso escolar que no le permitieron contestar la entrevista.

\section{Técnica de recogida de información}

Se utiliza como técnica de recogida de datos la entrevista en profundidad. La entrevista en investigación cualitativa permite obtener información respecto a un tema en específico en base a las experiencias del entrevistado (Vargas, 2012). Se utilizó la entrevista semi-estructurada debido a sus características, entre ellas su informalidad y su flexibilidad, la que le permite adaptarse a los sujetos participantes y a las condiciones de recolección de datos en el campo. La entrevista fue guiada por una pauta previamente elaborada por la investigadora, como una forma de direccionar la investigación para el cumplimiento de objetivos. 
En cuanto a la cantidad de participantes, se determinó por saturación teórica, es decir, cuando la información obtenida no aportó nuevos datos para el objetivo de la investigación (Ortiz, 2015). Cabe señalar que para efectos de la investigación cualitativa la saturación teórica no se logra por la cantidad de entrevistados, sino por la riqueza de sus entrevistas y la información que entregan (Nicolini, 2015).

\section{Selección de los contextos}

La presente investigación se lleva a cabo en un colegio de la comuna de Concepción. El índice de vulnerabilidad del colegio (IVE-SINAE) corresponde a 93,10\% (JUNAEB, 2018), por tanto, gran parte de los estudiantes se encuentra en una situación vulnerable.

El establecimiento educacional presenta dos niveles: Educación Parvularia y Enseñanza Básica, con sus respectivos cursos. Dentro de la formación, se considera la dupla psicosocial encargada de la convivencia escolar para resolver situaciones o problemas que pudiesen presentar los estudiantes. Los cursos seleccionados son séptimo y octavo básico, puesto que en esos cursos es posible encontrar adolescentes de 13 años, que de acuerdo con Sánchez y Cerezo (2010), en esa edad se presenta con mayor frecuencia e intensidad el acoso escolar.

\section{Trabajo de campo}

En cuanto al acercamiento al establecimiento educacional, en el mes de octubre (2018) se lleva a cabo la primera reunión con la trabajadora social del colegio para entregar información sobre la investigación, es decir, describir los participantes, los objetivos y el tiempo estimado en el cual se llevaría a cabo, para que posteriormente, ella informase al director del establecimiento educacional. Una vez realizada la coordinación, la trabajadora social se pone en contacto con la investigadora para informar que la investigación fue aceptada por el director.

Durante el mes de noviembre (2018) se lleva a cabo nueva coordinación para determinar la cantidad de adolescentes que poseen 13 años, una vez determinado el número exacto (22) se realiza entrega de 
los consentimientos informados a la trabajadora social para que se los envíe a los padres por medio de los estudiantes. Estos no son entregados en reunión de padres porque existe baja asistencia a estos eventos. Cabe señalar que esta fue una de las mayores dificultades, ya que los/ as adolescentes no entregaban los consentimientos a los padres, aun cuando se les hizo entrega más de una vez. Luego de este período, se coordinaron días y horarios para que la investigadora realizara las entrevistas a los/as estudiantes que habían sido autorizados por los padres.

Respecto a los participantes, de acuerdo con lo señalado por la trabajadora social, poseen un perfil parecido, destacándose entre sus compañeros por ser participativos y demostrar buena conducta.

Se llevó a cabo un análisis de contenido, de acuerdo con lo que plantea Abela (2008), este análisis es una técnica de interpretación de textos, ya sean escritos o grabados, basada en la lectura (textual o visual) como instrumento de recogida de información, lectura que debe ser realizada siguiendo el método científico, es decir, debe ser sistemática, objetiva replicable y valida. A diferencia de otras técnicas, mezcla la interpretación con el análisis de datos, por ello fue seleccionada.

En primer lugar, se lleva a cabo la transcripción textual de entrevistas, para ellos se utiliza normas según Jefferson, luego se prepararán los datos para el análisis, posteriormente se lleva a cabo la codificación de las unidades de análisis, luego se determinan las categorías y la codificación de estas para dar paso a los hallazgos, para este caso se realiza a través de categorías (dimensiones) previamente establecidas en la entrevista cualitativa.

El plan de análisis se realiza con ayuda de CAQDAS (ComputerAided Qualitative Data Analysis), es decir, software de análisis cualitativo de datos. Dentro de los tipos de software se encuentran los que permiten realizar análisis de contenido, dividir el texto en segmentos y que se les asigne un código, de esta forma se da continuación a la construcción teórica, para ello se utiliza el programa ATLAS-ti v.8 (Caro y Díez, 2005). 


\section{Resultados}

\subsection{Experiencia de bullying}

De acuerdo al relato de los/as entrevistados/as, el bullying es un fenómeno que se encuentra presente en el establecimiento educacional. Como interpretación de sus narraciones, es posible identificar cierta conciencia respecto al fenómeno, pero de manera superficial, es decir, no existe un proceso reflexivo real y de comprensión respecto a lo que involucra e implica este fenómeno en sus participantes, sean directos o indirectos, situación que ocasiona que los/as entrevistados/as no sepan situarse dentro del fenómeno, por ello desconocen lo que pueden lograr con sus intervenciones.

Respecto a los tipos de bullying, a los/as participantes se les dificulta identificarlos, ya que engloban todas las manifestaciones en un solo término: bullying. Para ellos, no existirían grados de daño entre los tipos de acoso escolar, al contrario, todos cumplirían el mismo objetivo, es decir, generar un efecto nocivo o negativo en la víctima, pero de diferentes formas, mediante golpes, insultos u otro mecanismo. Entre las formas de bullying más utilizadas, se encuentra el acoso verbal porque no deja marcas visibles y el acoso físico, este último se asociaría a niños/as más pequeños, debido a la impulsividad y a la falta de herramientas para resolver conflictos.

Respecto a las consecuencias del acoso escolar, los/as participantes logran reconocer las consecuencias en las víctimas tales como depresión, ausentismo escolar, aislación, suicidio, entre otras; sin embargo, en los demás roles (victimario y observador) no perciben mayores consecuencias, aparte de la culpa en los/as observadores/as y gratificación por parte del victimario, por lo tanto, esta carencia de información respecto a los efectos que genera el acoso escolar en todos sus roles, se convierte en un aspecto que debe ser trabajado con los estudiantes para que comprendan la magnitud del fenómeno.

Al hablar de experiencias de bullying, se aprecian diferencias en la forma de reaccionar de los/as entrevistados/as cuando se trata de un cercano. Estos actúan defendiendo al victimario, debido al lazo que los une; no obstante, cuando se trata de un compañero con el cual 
no poseen un vínculo estrecho, se abstienen por falta de interés y en algunos casos por miedo a las consecuencias, lo cual demostraría la importancia de fortalecer las relaciones entre compañeros/as.

\subsection{Mecanismos de acción observadores/as}

Entre los mecanismos de acción se distinguen tres roles, esto es, observador/a activo, pasivo y proactivo. La motivación para desarrollar el rol proactivo se relaciona a la compasión y simpatía por la víctima y en algunos casos al logro de empatizar con la situación, los/as estudiantes defienden a la víctima, increpando al agresor o informando a un/a profesor/a para que la situación se detenga. En el caso del observador activo, demuestra inmadurez emocional, al considerar las agresiones una broma, sin tener en cuenta el dolor o humillación que vive la persona agredida, por ello apoya al agresor con risas u otros mecanismos. En el caso del observador pasivo, se aprecia individualidad en este rol, ya que su falta de intervención se vincula al poco interés y en algunos casos el temor a las consecuencias.

El significado entregado a la experiencia de observador/a responde a la acción que desarrollaron. En el caso del observador/a pasivo, no demuestra mayor conciencia respecto al significado de su acción en el hecho observado, para ellos/as resulta más importante evitar el riesgo de involucrarse, privilegiando la autoprotección, frente al riesgo de asumir las consecuencias de defender a la víctima. En cuanto al observador proactivo, no solo actúa con la intención de defender a la víctima, también visualiza ciertas ventajas en su acción, como la posibilidad de tener un respaldo si es que llegase a ser agredido.

Respecto a la participación del observador en la dinámica de bullying, se presentan dos puntos de vista en la investigación; en primer lugar, se considera que la intervención como observador/a no genera mayores cambios en la dinámica, porque los/as agresores/as mantienen la conducta, por lo tanto, aun cuando se recurra al diálogo o se denuncien los hechos, el bullying continuará. Por otro lado, existe una postura más optimista respecto a la acción del observador/a, en la que el interrumpir el acoso permite que la víctima deje de sufrir. No obstante, en la última postura se detecta escaso proceso reflexivo, respecto 
a la intervención en la dinámica, ya que, si bien la agresión se detiene en el momento, nada asegura que no vuelva a ocurrir.

\subsection{Clima escolar}

Los/as entrevistados conciben el clima escolar como un ambiente protector, por ello, en muchas ocasiones le atribuyen una perspectiva familiar, sobre todo al grupo (curso), sin embargo, este ambiente protector presenta falencias en aspectos comunicacionales, entre padres, profesores/as, personal administrativo y estudiantes, ya que muchas de las situaciones conflictivas que se presentan no son informadas. Por otro lado, se detectan falencias en las técnicas de resolución de conflictos, demostrando en más de una ocasión poca eficacia en los resultados. Estos factores, si bien perjudican el clima escolar, no han sido trascendentales para alterar la percepción de los/as participantes.

En cuanto al bullying, cumple un rol relacional dentro del clima escolar, es decir, es una forma negativa de relacionarse, que genera efectos en todos aquellos que se encuentran a su alrededor, debido a esta característica resulta difícil de abordar porque implicaría cambiar la forma de relacionarse de los estudiantes. Por otro lado, resulta necesario generar instancias dentro del establecimiento educacional para que los estudiantes puedan denunciar las situaciones de acoso escolar que detectan, de forma anónima, ya que, si bien los casos son abordados en la hora de orientación, esta instancia no genera la confianza suficiente para que los estudiantes hablen.

\subsection{Aspectos culturales}

En la investigación se perciben dos sesgos culturales: la familia y los medios de comunicación. En relación a la familia y la temática bullying, en general se presentan dos posturas, esto es, familias abiertas a hablar sobre la temática y aquellas donde es un tema tabú, debido a historias familiares en las cuales integrantes del grupo lo vivieron. En este punto, resulta necesario tener una visión crítica, ya que evitar hablar sobre un tema, no lo elimina, por el contrario, aumenta la sensación de miedo, perjudicando los canales de comunicación, es decir, 
cuando un fenómeno se vuelve tabú en el grupo familiar, las posibilidades de obtener nueva información sobre él, son mínimas, por lo tanto, si volviese a ocurrir un hecho de acoso escolar, tal vez el/a adolescente no tendría la confianza necesaria para revelar lo ocurrido, puesto que dentro del hogar no está la instancia o espacio para hablar sobre él.

El segundo sesgo cultural percibido son los medios de comunicación. De acuerdo con los/as entrevistados, estos medios no generan mayor cambio o efecto en sus opiniones o acciones; sin embargo, durante las entrevistas fue posible identificar algunas influencias en los mecanismos de acción por parte de quienes defendieron a la víctima, ya que los programas que visualizaban fomentaban ese tipo de prácticas. Por otro lado, respecto a los medios de comunicación, los/ as entrevistados solo hicieron referencia a la televisión, destacando información entregada sobre el fenómeno, como los casos que han terminado en suicidio. Lo relevante de este último punto es que una de las razones por la cual el bullying es considerado un fenómeno dañino socialmente es por los casos terminados en muerte; por lo tanto, aun cuando los/as entrevistados plantean que este medio no genera un impacto en la gente, la realidad es que la difusión de esa información permite que las personas sean un poco más conscientes respecto al fenómeno y sus efectos.

\section{Discusión y conclusiones}

Uno de los hallazgos de la investigación responde al posicionamiento de los/as estudiantes dentro del fenómeno de investigación, es decir, estos aún no logran determinar cuál es su lugar en la dinámica, así como tampoco vislumbran el poder que tienen sus acciones para impedir el acoso, por ello se observa inconsistencia en los mecanismos de acción desarrollados. Las razones por las cuales los/as observadores/ as no intervienen es porque tienen miedo a convertirse en víctimas y porque piensan que la podrían perjudicar (Hazler, 1996); sin embargo, en la presente investigación se presentaron otras razones como el individualismo, ya que en más de una ocasión los/as participantes ase- 
guraron que la falta de intervención se debía al poco interés, es decir, cómo la situación no los afectaba o no consideraban necesario intervenir. Respecto al factor miedo, en los casos donde los estudiantes eran de cursos superiores, influyó en la conducta el temor a ser agredidos, sin embargo, esta no es una constante, ya que no todos los casos son protagonizados por estudiantes de mayor edad.

Sumado a lo anterior, se encuentra la normalización de prácticas violentas en actividades recreacionales que realizan diariamente. En las narraciones de los estudiantes es posible verificar cómo relatan con normalidad juegos un tanto agresivos, que para ellos solo responden a una forma de expresión y diversión, obviando qué representan esos juegos para los/as demás involucrados/as. Autores como Musalem y Castro (2015) reafirman lo anterior, señalando que aun cuando el bullying puede partir como una broma, este escapa de cualquier tipo de juego, ya que posee la intencionalidad del agresor de generar daño a la víctima.

En general, se percibe que los/as participantes han aprendido a lidiar con el bullying es su vida escolar, convirtiéndose incluso en un proceso mecánico del que en algunos casos no son conscientes, como por ejemplo; cuando tuvieron que describir los tipos de acoso que identificaban, estos no lo lograron en una primera instancia, posteriormente hubo que profundizar en la problemática para que realizaran una distinción entre las manifestaciones de acoso escolar que se presentaban entre los/as estudiantes, lo que habla de una falta de problematización del fenómeno, aspectos que de una u otra forma contribuyen en su continuidad.

Respecto al clima escolar, los/as participantes lo perciben como un espacio de desarrollo en donde es posible establecer lazos, amistades y vínculos perdurables, no obstante, este espacio no es perfecto, presenta algunas fallas como el bullying y los problemas comunicacionales que fortalecen su presencia, generando preocupación entre los/as entrevistados y en algunos casos resignación.

Llama la atención dentro de los resultados de la investigación, la lealtad que sienten los/as entrevistados/as hacia compañeros/as y el colegio en general, de hecho se percibe una especie de incomodidad en estos cuando se habla de conflictos, demostrando inseguridad al 
momento de informar los problemas que se presentan, respondiendo a códigos presentes en los establecimientos educacionales que no son enseñados de forma directa, pero que de todas formas son entendidos por los/as estudiantes, al punto de condicionar en alguna medida su forma de actuar.

En cuanto al punto aspectos culturales, destaca la familia y los medios de comunicación. En el caso de la familia, se perciben algunas influencias en la forma de actuar de los entrevistados, ya que aun cuando señalaron hechos en los cuales defendieron a la víctima, en la mayoría de los casos no tuvieron la misma reacción, es decir, han desarrollado el rol de observador/a pasivo/a, lo que se condice con las recomendaciones de los padres, quienes sugirieron evitar conflictos como el bullying con la finalidad de impedir consecuencias que los puedan dañar. Si bien se percibe en las sugerencias entregadas, un aspecto protector en necesario hacer énfasis en el rol que cumplen los/as estudiantes dentro del establecimiento educacional, es decir, a ellos/as también les corresponde garantizar relaciones saludables que favorezcan el clima escolar, esto no será logrado si la mayoría no es capaz de empatizar con el sufrimiento de otros/as, siendo fundamental contar con el apoyo y respaldo de la familia, por lo tanto, es un aspecto que debe ser trabajado.

En relación a los medios de comunicación, los/as individuos no son conscientes de las influencias que reciben, sin embargo, estas de alguna u otra forman causan un efecto en sus vidas. En el caso de un fenómeno tan complejo como el acoso escolar, los/as participantes consideran que debería existir mayor información para comprender la profundidad de este y el alcance que tiene, estas sugerencias nacen de la importancia que le otorgan al bullying, para ellos/as es necesario hablar de él porque consideran que socialmente no ha causado el impacto necesario.

Desde los/as propias participantes se aprecian algunas propuestas que podrían ser claves al momento de la intervención por parte de los establecimientos educacionales como charlas periódicas y de calidad. Esto se condice con las experiencias internacionales como el programa KIVA y el WiSk. Es importante abordar temas como las habilidades sociales, temas culturales, diversidad, inclusión, resolución de conflic- 
tos, empatía, trabajo en equipo y muy importante el conocimiento $\mathrm{del} / \mathrm{a}$ otro/a, ya que este punto permitirá el posicionamiento emocional en el otro en la relación de acoso y en los mismos observadores, como agentes de cambio y detención de la violencia escolar y como los propios participantes sugieren, estas temáticas deben ser trabajadas desde ellos/as, a especie de tutorización entre pares e incluso aquellos/ as que cometen actos de acoso, debiesen ser parte de estos tópicos. Además, sugieren que se aporte en un cambio cultural en la aplicación de sanciones respecto de acciones de acoso, ya que en los colegios hoy se utiliza la expulsión como norma sancionatoria, pero los participantes de este estudio sugieren otras formas de aplicar la norma, como el trabajo comunitario dentro del colegio (Limpiar, ordenar, apoyar en labores cotidianas). Anidado a ese cambio, se propone que se les informe a los padres y madres sobre el bullying y los daños que se provocan y así incentivar procesos reflexivos en los estudiantes y sus familiares.

Desde las experiencias internacionales, se destaca un trabajo en conjunto de toda la comunidad educativa, donde la reparación a la víctima es clave, pero también debe existir un proceso de "reparación" en el/a agresor/a, ya que han aprendido como normal las conductas violentas, por lo tanto, hay que deconstruir ese tipo de actos como comunidad educativa y no como intervenciones psicosociales aisladas, dichos elementos generalizados y reflexionados a nivel institucional permitirá un avance, en primera instancia a la visibilización del fenómeno y, en segunda, a trabajar en procesos y estrategias para su prevención y reparación del daño que se puede causar.

\section{Referencias}

Abela, J. (2008). Las técnicas de análisis de contenido: Una revisión actualizada. Granada (España). Universidad de Granada. Recuperado de: http://mastor.cl/blog/wp-content/uploads/2018/02/Andreu.-analisisde-contenido.-34-pags-pdf.pdf

Arango, A. (2014). El bullying y la relación entre los implicados (victimas, victimarios y espectadores). Tesis de Licenciatura para optar al grado de psicologa, Universidad de la Sabana. Recuperado de: https://intellectum. 
unisabana.edu.co/bitstream/handle/10818/11372/Alexandra\%20Arango\%20\%28tesis\%29.pdf?sequence=1\&isAllowed=y

Arroyave, P. (2012). Factores de vulnerabilidad y riesgo asociados al bullying. Revista CES Psicología, 5, 118-125.

Azofra, A. (2017). Acoso escolar en Educación Primaria: KIVA y otros nuevos métodos de abordarlo. Tesis para optar al grado de Educación Primaria. Universidad de la Rioja, España. Recuperado de: https://biblioteca. unirioja.es/tfe_e/TFE002374.pdf

Bandura, A (1976). Teoria del aprendizaje social. Madrid: Editorial Espasa Calpe.

Briceño, A. V. (2017). Relación entre disfunción familiar y conductas de agresión entre pares: Colegio Municipal Nueve de Octubre. Tesis para optar al grado de Psicólogo. Universidad Central de Ecuador. Recuperado de: http://www.dspace.uce.edu.ec/bitstream/25000/10276/1/TUCE-0007-PC004-2017.pdf

Bronfenbrenner, U. (1987). La ecología del desarrollo humano. Experimentos en entornos naturales y diseñados. Barcelona: Paidós.

Caro, F. y Díez, E. (2005). Investigación cualitativa asistida por ordenador en economía de la empresa en: Revista de Investigaciones Europeas de Dirección y Economía de la Empresa, 11,45-58.

Cuevas, M., y Marmolejo, M. (2016). Observadores: un rol determinante en el acoso escolar. Pensamiento Psicológico, 14, 89-102.

Galeano, M. E. (2004). Diseño de proyectos en la investigación cualitativa. Medellín: Fondo Editorial Universidad Eafit.

García, M., y Ascencio, C. (2015). Bullying y violencia escolar: Diferentes similitudes, actores, consecuencias y origen. Revista Intercontinental de Psicología y Educación, 17, 9-38.

Gifre, M. y Guitart, M. (2013). Consideraciones educativas de la perspectiva ecológica de Urie Bronfenbrenner. Contextos educativos. Revista de Educación, 15, 79-92.

González, V., Mariaca J. y Arias J. (2014). Estudio exploratorio del bullying en Medellín. Pensando Psicología, 10, 17-25.

Hazler, R. J. (1996). Breaking the cycle of violence: Interventions for bullying and victimization. Washington: Accelerated Development.

JUNAEB (2018). Prioridades con IVE SINAE básica, media y comunal. Recuperado de: www.junaeb.c1/wp-content/.../IVE-POR-RBD-BASICAMEDIA-COMUNA-2018.xlsx

KivaProgram. (2013). Programa Kiva para el acoso escolar bullying guía 
para padres. Recuperado de http://www.kivaprogram.net/assets/files/ Spanish\%20KiVa\%20Parents\%20Guide.pdf.

López, G., y Guiamara, Y. (2016). La escuela: espacio de encuentro y armonía o escenario de violencia. Ixaya. Revista Universitaria de Desarrollo Social, 10, 89-124.

Mäkelä, T. y Catalán, B. (2018). Programa de convivencia y anti-acoso escolar KiVa: Impacto y reflexión. Anales de la Fundación Canis Majoris, 2, 234-258.

Musalem, B. y Castro, O. (2015). Qué se sabe de bullying. Revista médica clinica Las Condes, 26, 14-23.

Nicolini, C. (2015). Informe: El Análisis de Contenido como Técnica de Investigación. Utilización del software ATLAS.TI. Dirección de Estudios. Innovación Curricular y Desarrollo Docente. Universidad de Mejoramiento Docente. Recuperado de: http://www.upla.cl/innovacioncurricular/wp-content/uploads/2013/06/Informe-Final-AT-14_-CamiloNicolini.pdf

Olweus, D. (1993). Acoso escolar, "bullying", en las escuelas: hechos e intervenciones. Recuperado de: http://convivejoven.semsys.itesi.edu.mx/ cargas/Articulos/ACOSO\%20ESCOLAR\%20BULLYING\%2C\%20EN\%20 LAS\%20ESCUELAS-HECHOS\%20E\%20INTERVENCIONES.pdf

Otzen, T., y Manterola, C. (2017). Técnicas de Muestreo sobre una Población a Estudio. Revista Internacional de Morfología, 35, 227-232.

Ortega, R., Del Rey, R.d. y Casas, J.A. (2013). La convivencia escolar: clave en la predicción del bullying. Revista Iberoamericana de Evaluación Educativa, 6, 91-102.

Ortiz, G. (2015). La entrevista cualitativa. Técnicas de Investigación Cuantitativas y Cualitativas. Recuperado de: https://rua.ua.es/dspace/bitstream/10045/47795/1/Tema\%206\%20La\%20Entrevista\%20Cualitativa\%20Grado\%202014-15.pdf.

Pulido, L. y Tarancón, P. (2018). Opiniones y experiencias respecto al bullying: estudio cualitativo en un Instituto de Educación Secundaria de la ciudad de Albacete. Ensayos. Revista de la Facultad de Educación de Albacete, 33, 1-17.

Romero, S., y Bravo, V. (2017). El bullying y las consecuencias emocionales en los victimarios. Tesis de Licenciatura en Psicología. Universidad Estatal de Milagro. Recuperado de: http://repositorio.unemi.edu.ec/ bitstream/123456789/3582/1/EL\%20BULLYING\%20Y\%20LAS\%20CONSECUENCIAS\%20EMOCIONALES\%20EN\%20LOS\%20VICTIMARIOS. DOCX\%20PDF.PDF 
Romera, E. M., Ortega, R., Rey, R.D., Casas, J. A., Viejo, C. M., Gómez, O., y Luque, R. (2017). Bullying, cyberbullying y dating violence: Estudio de la gestión de la vida social en estudiantes de Primaria y Secundaria de Andalucía. Sevilla: Fundación Pública Andaluza.

Ruiz, R., Riuró, M. y Tesouro, M. (2015). Estudio del bullying en el ciclo superior de primaria. Educación XXI, 18, 345-368.

Salmivalli, C. (1999). Participant role approach to school bullying: implications for intervention. Journal of Adolescence, 22, 453-459.

Sánchez C., y Cerezo F. (2010). Variables personales y sociales relacionadas con la dinámica bullying en escolares de Educación Primaria. Revista Electrónica de Educación Psicoeducativa, 8, 1015-1032.

Sánchez, P. (2013). El bullying como construcción social, más allá de las víctimas, los agresores y los testigos. La familia, los docentes y la sociedad. Revista Latinoamericana de Estudios de Familia, 15, 222-247.

Spiel, C., Strohmeier, D., y Atria, M. (2010). Evaluación de programas de intervención: En proyecto "Beispiel des WiSK". En R. Ortega (ed.). Agresividad injustificada, bullying y violencia escolar (pp. 385-412). Madrid: Alianza.

Vargas, I. (2012). La entrevista en la investigación cualitativa: nuevas tendencias y retos. Revista Electrónica Calidad en la Educación Superior, 3,119139. 